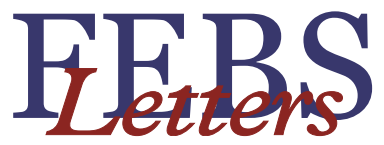

journal homepage: www.FEBSLetters.org

Review

\title{
Challenges in quantitative single molecule localization microscopy
}

\author{
A. Shivanandan, H. Deschout, M. Scarselli ${ }^{1}$, A. Radenovic* \\ Laboratory of Nanoscale Biology, Institute of Bioengineering, School of Engineering, EPFL, Lausanne, Switzerland
}

\section{A R T I C L E I N F O}

Article history:

Received 1 May 2014

Revised 1 June 2014

Accepted 2 June 2014

Available online $\mathrm{xxx}$

Edited by Elias M. Puchner, Bo Huang,

Hermann E. Gaub and Wilhelm Just

\section{Keywords:}

Single molecule localization microscopy

SMLM

Photo Activated Localization Microscopy

PALM

Single molecule

Fluorescent proteins

Co-localization

Cluster analysis

Quantitative SMLM

\begin{abstract}
A B S T R A C T
Single molecule localization microscopy (SMLM), which can provide up to an order of magnitude improvement in spatial resolution over conventional fluorescence microscopy, has the potential to be a highly useful tool for quantitative biological experiments. It has already been used for this purpose in varied fields in biology, ranging from molecular biology to neuroscience. In this review article, we briefly review the applications of SMLM in quantitative biology, and also the challenges involved and some of the solutions that have been proposed. Due to its advantages in labeling specificity and the relatively low overcounting caused by photoblinking when photo-activable fluorescent proteins (PA-FPs) are used as labels, we focus specifically on Photo-Activated Localization Microscopy (PALM), even though the ideas presented might be applicable to SMLM in general. Also, we focus on the following three quantitative measurements: single molecule counting, analysis of protein spatial distribution heterogeneity and co-localization analysis.
\end{abstract}

() 2014 Federation of European Biochemical Societies. Published by Elsevier B.V. All rights reserved.

\section{Introduction}

With the invention of single molecule localization microscopy (SMLM) [1-3], it has become possible to extend the advantages of fluorescence microscopy beyond its diffraction limited spatial resolution of about $200 \mathrm{~nm}$. This provides the possibility of resolving organelles or even single molecules with an order of magnitude better resolution, in multiple color channels and in 2D as well as 3D. Recent reviews on the updates on the technology and its uses can be found in $[4,5]$. SMLM can potentially be used for quantitative measurements [6,7], e.g., in counting the number of molecules of a protein specie [8] and stoichiometry estimation of protein complexes [9-11], characterizing the spatial distribution of a protein specie [12-15], estimating the co-localization or co-clustering between organelles and also single molecules (SM) [16-19], estimating the relative positions of various components in a protein complex with high precision [20,21], and estimating the diffusion coefficients by means of single particle tracking (SPT) in a dense sample [22,23]. Two basic variants of SMLM are Photo-Activated Localization Microscopy (PALM) and STochastic Optical Reconstruction Microscopy

\footnotetext{
* Corresponding author.

E-mail address: aleksandra.radenovic@epfl.ch (A. Radenovic).

1 Current address: Department of Translational Research and New Technologies in Medicine and Surgery, University of Pisa, Pisa, Italy.
}

(STORM). The former uses fluorescent proteins for labeling (called photo-activable fluorescent proteins, PA-FPs), whereas the latter uses organic dyes. Since the usage of fusion proteins used in PALM provides comparatively high specificity labeling as against immunolabeling (the typical labeling technique used for STORM), and since the phenomenon of photoblinking for PA-FPs is minimal (as against the photo-switchable organic dyes used in STORM, which typically blink 10 times or more before irreversible photobleaching [24]), PALM appears to be better suited for quantitative studies, and for this reason forms the focus of this article even though many of the ideas presented are applicable to SMLM in general. Yet, quantitative analysis with PALM is plagued by several sources of errors $[7,70]$, including that of a limited detection efficiency of label molecules in the range of $40-60 \%[16,25]$, a localization uncertainty in the order of $20-50 \mathrm{~nm}[26,27]$, overcounting in the range of $100 \%$ due to reappearance of label molecules due to photoblinking [15,28-30], errors in labeling, a sample drift in the order of $50-100 \mathrm{~nm}[1,31]$ and in the case of multi-color imaging, registration errors [16].

This review is divided into two parts. The application of SMLM has brought new discoveries in varied biological fields such as cell biology, neuroscience, microbiology and molecular genetics. First, we provide a bird's eye view of the applications of quantitative SMLM in these fields, focusing on the biological perspective. Then, with the help of cartoon figures, we explore in detail the challenges 
that are present in the use of SMLM, and specifically PALM, for quantitative experiments. We focus specifically on three specific quantitative applications of PALM: single molecule counting, analysis of protein spatial distribution heterogeneity and co-localization analysis. We also provide a brief summary of the methods that have been presented in the field to resolve the challenges presented.

\section{Quantitative SMLM and biology}

The possibility to quantify the number of proteins within biological assemblies and to characterize the protein spatial distribution has permitted to determine protein stoichiometry and distribution in signaling complexes. As a demonstration of this aspect, some groups, including ours, have analyzed the existence of protein aggregates such as oligomers and clusters for signaling receptors as $\mathrm{G}$ protein-coupled receptors (GPCRs), asialoglycoprotein receptors and RAS signaling molecules $[9,10,32]$.

For the $\beta 2$ adrenergic receptors, by means of quantitative cluster analysis, we found that the receptors are partially organized in mini-clusters only in the cardiomyocytes like-cells but not in other cell lines, and these oligomers are not lipid raft related but depend on actin cytoskeleton integrity (Fig. 1) [33]. Importantly, this conclusion was quite different from a similar report that was obtained using a different method named near-field scanning optical microscopy (NSOM) [71], as a demonstration of a better precision of PALM over other techniques. Receptor oligomers were not affected by the addition of different ligands, indicating that the receptor is already pre-associated before activation and is not related to receptor basal activity. In contrast, in a study by Renz et al. that made use of quantitative single molecule counting, it was shown that assembly of asialoglycoprotein receptors into homo- and hetero-oligomeric structures is dictated by exogenous ligands leading to the internalization of one receptor complex over another complex [9]. In this case, the authors used quantitative PALM together with ensemble Förster Resonance Energy Transfer (FRET) imaging. This experimental strategy has demonstrated the strong synergy that exists between these two different techniques combining the powerful sensitivity of FRET to detect receptor proximity with the capability to obtain direct visualization of receptor oligomers with PALM. A similar approach was also successfully applied to study another strategic protein in the RAS signaling, named RAF [10]. By means of cluster analysis, the authors showed how RAF exists between an inactive monomeric state in the cytosol and a multimeric condition at the cell membrane when activated.
Together, these results confirmed the importance of dimers and oligomers formation in RAF signaling, even though the precise biological role of these different multimeric states is yet to be determined.

Another relevant consequence of the introduction of SMLM has been a better definition of biological structures in the nanometer range. This has been particularly true in the neuroscience field whereas the morphology of neurons composed by dendritic spines and synapses is not perfectly suitable for confocal microscopy. For example, imaging presynaptic and postsynaptic scaffolding proteins in glomeruli of the mouse olfactory bulb using STORM, Dani et al. showed distinct punctate patters that were not resolved by conventional fluorescence image [34]. They quantified various morphological parameters, and were able to distinguish the presynaptic Bassoon and postsynaptic Homer1 clusters. In this line of research, another group studied, by means of cluster analysis, the postsynaptic density (PSD) organization in live rat hippocampal neurons [35]. PALM was able to localize scaffolding nanodomains of PSD-95 enriched preferentially of AMPA receptors compared to NMDA receptors. This post-synaptic architecture could be relevant for the amplitude of postsynaptic currents, suggesting the mechanism of PSD in regulating the strength and plasticity of the glutamatergic transmission. For the optimization of cell morphology measurements in living cells using Single-Particle Tracking PALM, it has been shown using Monte-Carlo simulations how some technical parameters such as the length of the excitation pulse can influence the imaging of spine and spine neck morphology in living neurons, making them erroneously thinner when imaged using a longer excitation pulse [36].

SMLM was also applied to study exocytosis in different cell types, such as chromaffin cells. In PC12 cells, PALM was able to determine the size of clathrin coated pits during reuptake of vesicular acetylcholine transporters [37]. In contrast to what was found with confocal microscopy, Bar-On et al. used PALM to demonstrate that syntaxin 1 and SNAP-25 clusters have a weak co-localization in PC12 cells [38]. Additionally, PALM helped to establish that clustered SNARE proteins are not involved in large dense core vesicles (LDCV) in the fusion process [39].

Another field in biology that has received attention for SMLM is microbiology, in particular for the study of bacteria and viruses. Because of the size of these microorganisms, the super resolution methods are suitable for revealing the details of their sub-cellular structures. For example, Ptacin et al., studying the partitioning (Par) apparatus that guides centromere segregation, were able to
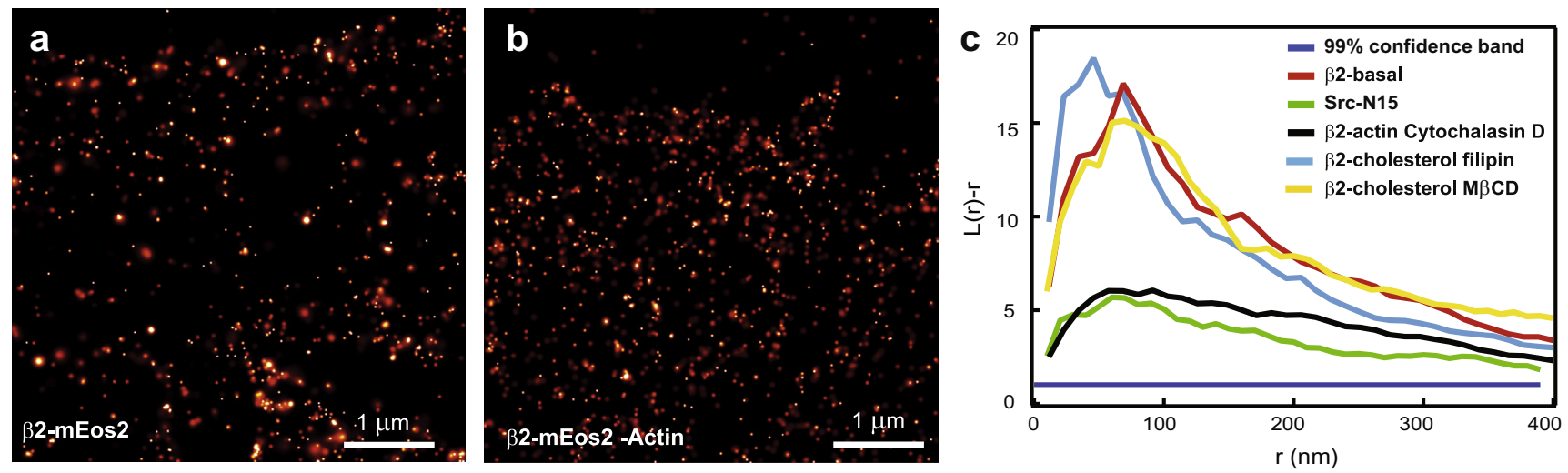





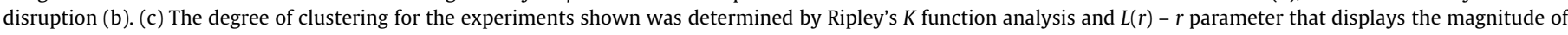

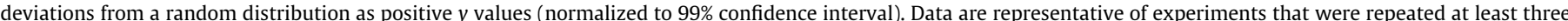


concentration of $5 \mathrm{mM}$, while actin microfilaments disruption was performed by preincubating for 30 min with cytochalasin $\mathrm{D}$ at the concentration of $2 \mu \mathrm{M}$. 
determine the ParA and ParB dynamics, suggesting that retracting ParA fibers are critical for this phenomenon [40]. SMLM also has shown its applicability in providing new details into viral infection. Using dSTORM, Pereira et al. were able to visualize and quantify the distribution of structural proteins of the human immunodeficiency virus type 1 (HIV-1) before and after infection of lymphoid cells [41]. Another work on the same theme was able to determine the distribution of the integrase enzyme (IN) of HIV in infected cells in the cytosol and in the nucleus, and to characterize its morphology [42]. The authors used a different version of the PALM technique, called FlAsH-PALM, where proteins are tagged with small tetracysteine motifs and the fluorescein arsenical helix binder.

Finally, super-resolution microscopies have opened a door in a deeper understanding of the chromosome organization and genome mapping. Wang et al. determined nucleoid-associated proteins distribution in live Escherichia coli cells [43], while another group was able to label 91 out of a total of 107 reference sites on a $180 \mathrm{~kb}$ human BAC gene with a 100 bp resolution [44]. DNA mapping with such resolution offers new potentials to uncover genetic variance and to facilitate medical diagnosis in genetic diseases.

\section{Challenges}

In this section, we review the critical challenges that are present in using SMLM, and specifically PALM, for quantitative measurements. While the challenges presented might be applicable to different types of quantification measures, we focus specifically on single molecule counting, analysis of protein spatial distribution heterogeneity and co-localization analysis. Before proceeding further, we note that it is possible that the image processing and localization algorithms used can also introduce errors in quantification, however this is treated in detail elsewhere $[45,46]$. A brief introduction to the quantitative measures being mentioned can be found in $[7,47]$.

\subsection{Labeling errors}

In SMLM, typically, the label tag can either be a fluorescent protein (FP) or an organic dye. In the former case, the labeling is usually done by means of overexpression, i.e. introduction to the cell of a plasmid with a vector consisting of the fusion of the sequences corresponding to the protein of interest and that of the fluorescent protein. In the latter case, the most typical labeling approach is immunolabeling, i.e., the dye is attached to the protein by means of antibodies. In this review we stick to PALM and hence labeling with PA-FPs, while noting that it is well known that the immunolabeling approach is more prone to labeling errors, including unspecific labeling.

In the case of labeling by means of overexpression, the protein expressed from the plasmid will exist in the cell along with those expressed endogenously. Since the latter is not fluorescent, this creates obvious limitations to quantification studies. One can get around this limitation by means of either knocking out the corresponding genes from the chromosome and thus making sure that only the overexpressed protein is present, or by introducing the fusion vector in the chromosome itself ("knock in"). In both cases, and especially the former, the effect on cell functioning might be profound.

It must be noted that it is the fluorescent tag that is imaged, and not the protein of interest itself. If the tag is a PA-FP, it typically has a size of about $4 \mathrm{~nm}$, and is attached to the protein of interest by means of an amino acid linker of length of up to $\sim 5 \mathrm{~nm}$ and hence the imaged structure can be off from the true structure by the vector sum of these quantities, in general in a random direction. This can introduce key errors in distance based studies, for example co-localization studies where the proteins of interest are physically co-localizing, whereas the FPs that label them might appear further apart, depending on their orientation within the labeled structure. Similarly, protein clusters will appear enlarged. Steric hindrance effects and label oligomerization can also complicate labeling accuracy. Also, in PALM experiments, typically a cell to be imaged is selected based on certain criteria - e.g. if the labeling is done properly, is sufficiently bright, and displays the expected morphology. This selection can be a source of sampling bias. High-throughput PALM that can image hundreds of cells at the same time offers a solution to this problem [72].

The impact of both incomplete labeling as well as the distance between the protein molecule of interest and the FP molecule is shown in Figs. 2a-b, 3a-b, and 4a-b for the three quantitative measures under discussion: counting, clustering and co-localization, respectively.

\subsection{Detection efficiency}

Since using FPs as label involves the complications associated with protein expression, errors in this step - misfolding, incomplete maturation etc. - can lead to the production of label molecules that are not fluorescent. Because of this reason, in the case of the conventional GFP, typically only $80 \%$ of the molecules that are present in the sample can be imaged [48]. In the case of PA-FPs, the fraction is even lower, due to incomplete photo-conversion. In the case of the relatively bright PA-FP mEOS2, this fraction is about $60 \%$, and for several other PA-FPs it can be as low as $40 \%[25,49]$.

This can directly affect counting studies, as the number of counted molecules can be underestimated by the same fraction (Fig. 2c). Other quantification measures might also be affected for example, in the case of SM co-localization, assuming that the used co-localization measure is linearly related to the detection efficiency, the co-localization will be underestimated by a fraction $x y$, where $x$ is the detection efficiency in one channel, and $y$ in the other, leading to an underestimate of as low as $20 \%$ for commonly used PA-FP pairs (Fig. 4c) [49]. However, in practice, the effect of limited detection efficiency on cluster (Fig. 3c) and co-localization analysis is not well explored.

It is possible to use the obtained count as a lower bound (after correcting for blinking artifacts) for the counting. In the specific case of identifying protein complex stoichiometry by means of counting the photobleaching steps, a model that accounted for detection efficiency by a binomial model was found to provide accurate results $[9,48,50]$. A similar approach, of incorporating the detection efficiency in a model for the ratio between monomers and dimers, has been reported [10]. Others have attempted to first characterize the relative detection efficiency of fusion protein pairs, and using it to estimate the stoichiometry for a target system [9].

\subsection{Localization uncertainty}

Each photon from the emitter molecule provides a sample of the PSF from the molecule. Based on these samples, single molecule localization algorithms provides an estimate for the position of the fluorescent molecule. This estimate is prone to uncertainties due to multiple reasons, predominated by limited sampling, i.e., by the limited number of photons obtained from the molecule. The resulting uncertainty in estimation can be quantified [26,27], and assuming a Gaussian model for the uncertainty, is known to mainly vary inversely proportional to the square root of photon count collected $(N)$, i.e., $\sigma_{l o c} \propto \frac{\sigma_{P S F}}{\sqrt{N}}$, where $\sigma_{l o c}$ is the standard deviation of the Gaussian uncertainty model, and $\sigma_{P S F}$ that of the Gaussian approximation of the point spread function. Since the photon count typically collected from a PA-FP molecule is less than 1000, the 

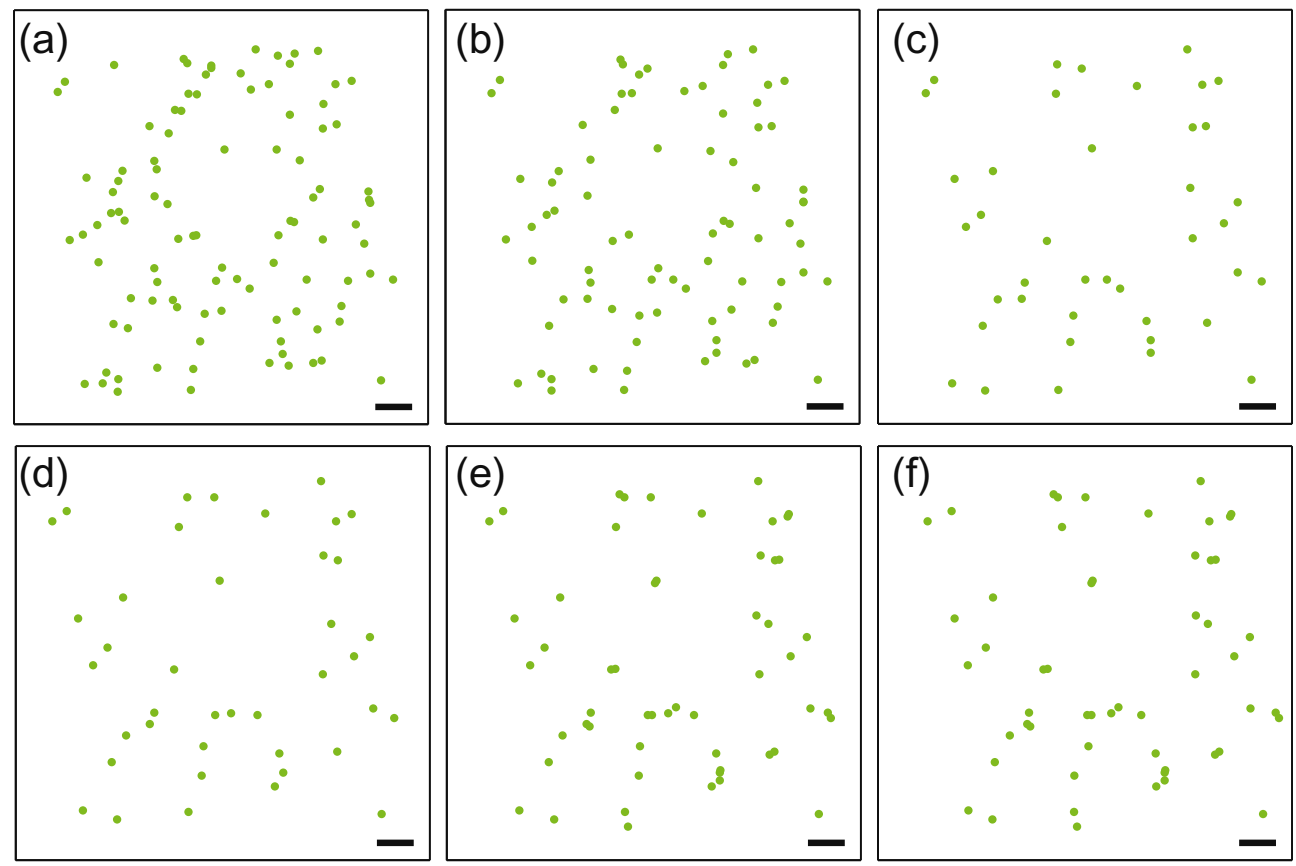

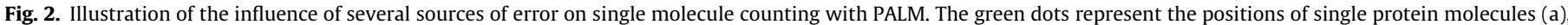

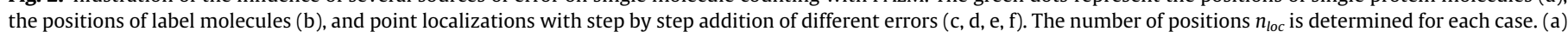

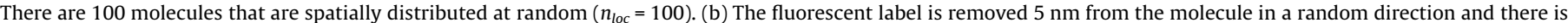

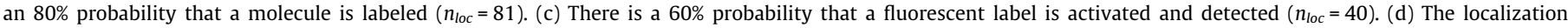

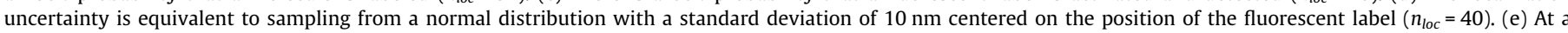

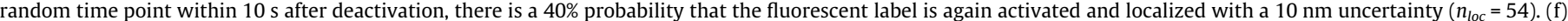

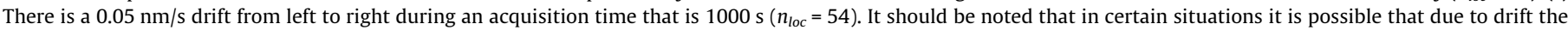
molecule count may be affected since the area of imaging changes over time. The scale bars represent $100 \mathrm{~nm}$.
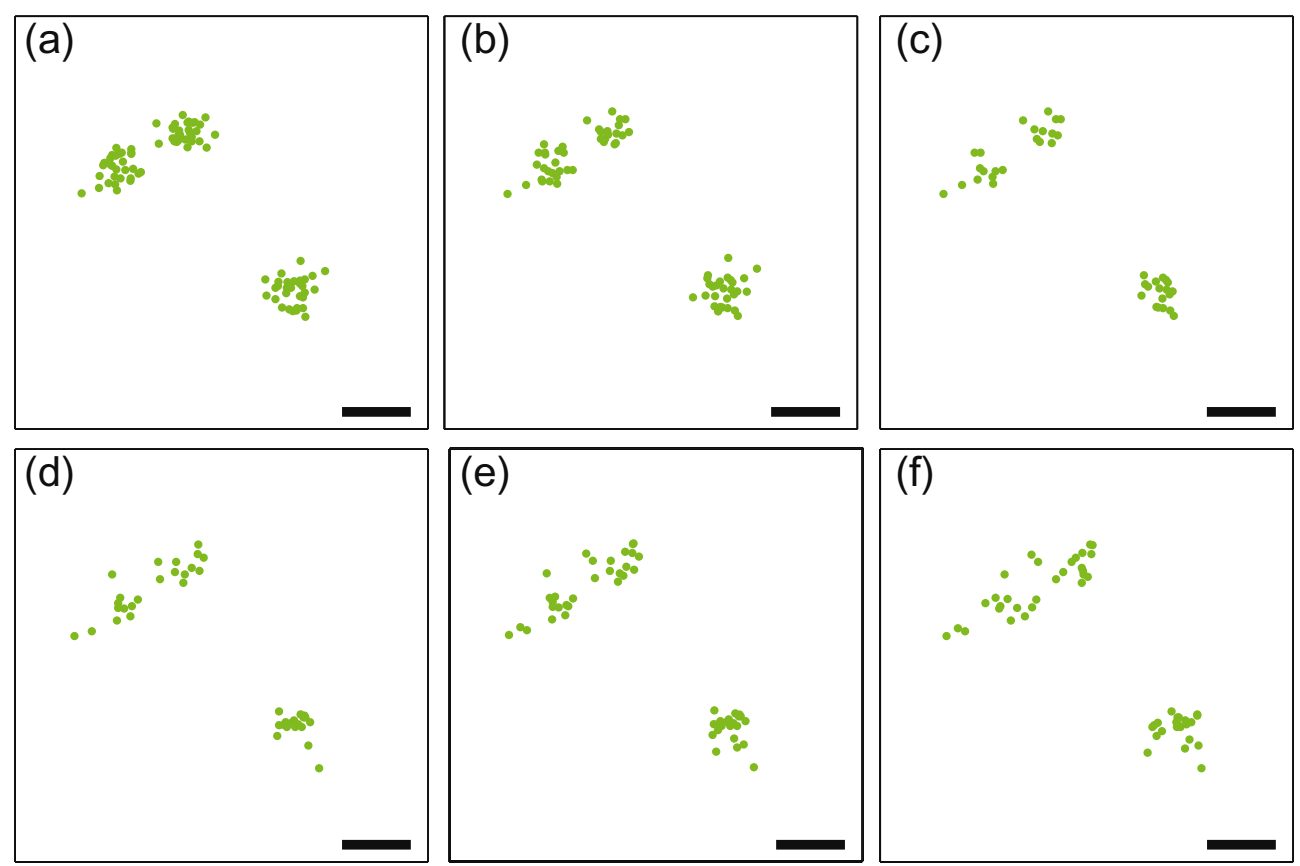

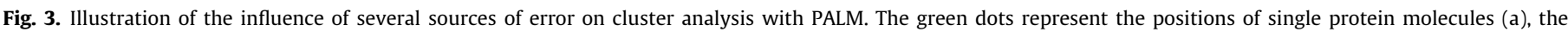

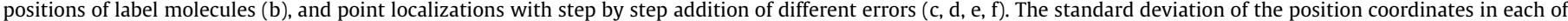



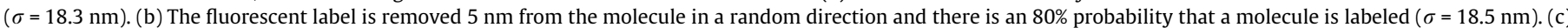

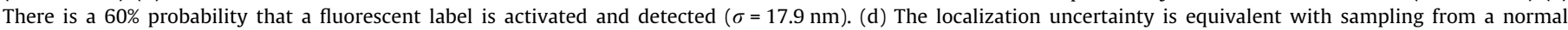

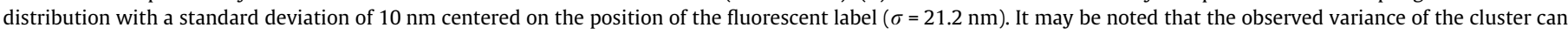

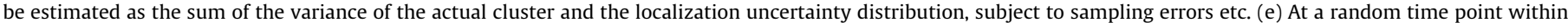

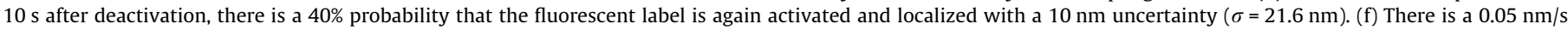
drift from left to right during an acquisition time that is $1000 \mathrm{~s}(\sigma=25.3 \mathrm{~nm})$. The scale bars represent $100 \mathrm{~nm}$. 

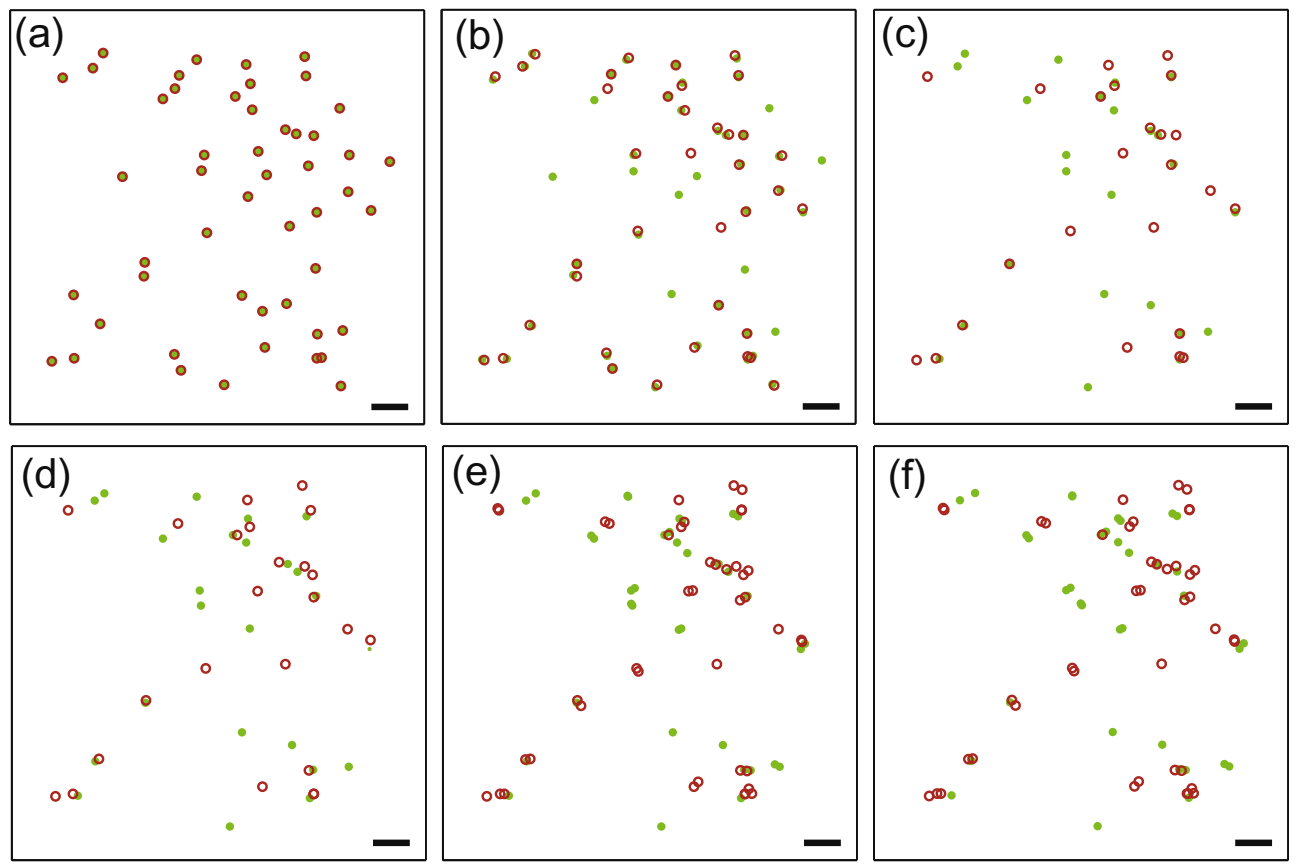

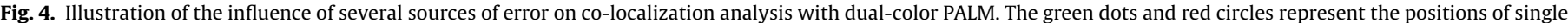

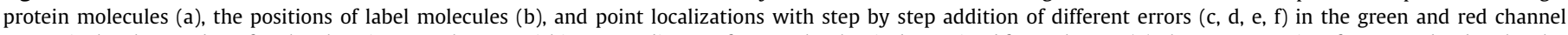



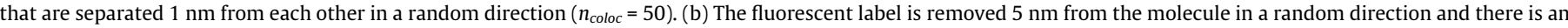

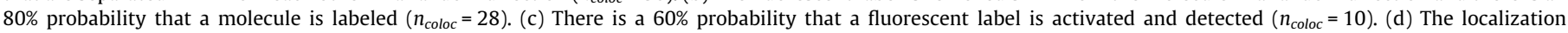

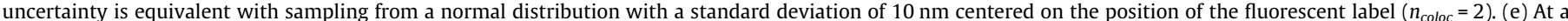

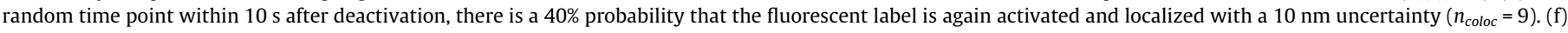

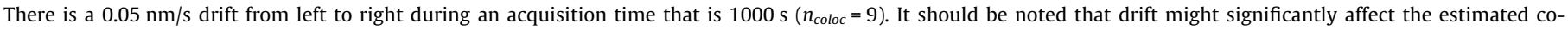
localization in many cases, unlike in this case. The scale bars represent $100 \mathrm{~nm}$.

precision $\sigma_{l o c}$ obtained is worse than $7 \mathrm{~nm}$ in the best cases, dropping to as low as $20 \mathrm{~nm}$ in practice, depending on the FP used, resulting in a full width half maximum (FWHM) resolution of $16-50 \mathrm{~nm}$. Also, the assumption of isometric emitters is not necessarily true in practice, and the errors resulting from dipole orientation can be up to $40 \mathrm{~nm}[45,51-53]$.

With careful imaging and analysis, i.e. by ensuring that the imaged molecules within a frame are spatially separated enough so that the localization algorithms can correctly identify them, it is possible to minimize the effect of localization uncertainty on counting measures (Fig. 2d). However, in the case of clustering and co-localization measures, this can cause a major impact (Figs. $3 \mathrm{~d}$ and $4 \mathrm{~d}$ ). For example, assuming that the localization estimation is approximately equivalent to sampling from a Gaussian distribution as mentioned above, centered at the true location of the emitter molecule, a cluster of molecules imaged in this way will appear enlarged, and hence the estimated cluster parameters will be affected (Fig. 3d).

If a Gaussian error model can describe the uncertainty in position localization, the estimated distance between two point localizations is described by a non-Gaussian distribution (specifically, a function of modified Bessel functions of order zero) [54]. If the true distance between two points is zero, and if the points are localized with an uncertainty as described above, then the estimated distance from the localizations will be greater than zero [55]. For example, if the localization precision for both points is $40 \mathrm{~nm}$ each, the estimated distance could be as high as $125 \mathrm{~nm}$ with a non-negligible probability [55]. Therefore, it is important to consider the effect of localization precision while performing quantitative analysis.

\subsection{Blinking}

The ideal fluorophore for counting will remain in the dark state until it is activated, and then will remain in the bright state emitting a large enough number of photons before photobleaching irreversibly, so that it does not reappear again in the bright state during imaging, resulting in overcounting. However, it has been observed that most available fluorescent proteins including GFP [56], and the PA-FPs [28,57], reappear after going to a long lived dark state from a bright state (called "blinking" behavior). Typical average values of reappearance is close to 1 for the commonly used fluorophore mEOS2, but it can be a few times higher for a significant fraction of imaged molecules. In either case it will result in overcounting (Fig. 3e), however if the molecule blinks multiple times it can also result in apparent clustering, forming artifacts amongst true physical clusters (Fig. 3e). Photoblinking artifacts can also lead to false positives in the case of co-localization, depending on the co-localization measure used (Fig. 4e).

It has been reported that the observed distribution for the time between the multiple appearances of a fluorophore (off time, $t_{\text {off }}$ ) can be fit to a single or double exponential distribution $[28,58,59]$. The average values of $t_{\text {off }}$ are comparatively on a much smaller scale compared to the imaging time of a PALM experiment, and hence on a spatial-temporal plot of the localizations, they appear as clusters. Therefore, by grouping together localizations that form these clusters, it is possible to correct for blinking artifacts [58-60]. Lando et al. used a Kalman filtering approach to correct for the artifacts [8], whereas Sengupta et al. have used a pair correlation based approach that is focused on the specific case of cluster analysis [15]. The latter approach works by separating the artifact clusters due to blinking from the true protein clusters by means of a model based on pair correlation function.

\subsection{Drift}

Since an SMLM experiment typically involves imaging 1000s of frames, due to temperature changes, air currents, mechanical 
vibrations etc., the sample might drift during the imaging time, in both lateral and axial direction. Since different subsets of molecules are imaged in different frames, drift can cause artifacts in SMLM imaging (Figs. 2f, 3f, and 4f). Basic efforts to minimize sources of drift, such as temperature stabilization and the use vibration-damping optical tables, are essential. Drift in the axial direction can be controlled by means of closed-loop feedback system that moves the sample stage in order to counteract the motion of sample [49,61]. Even then, lateral drift in the nanometer scale seems to be difficult to avoid (Figs. 2f, 3f and 4f) [49,62].

A common method to correct for sample drift is to insert fiducial markers in the sample, for example very bright fluorescent beads of $100 \mathrm{~nm}$ diameter [1]. Assuming that the fiducial marker does not move within the sample, and since the marker appears in all frames, the drift of the marker can be estimated, and the protein localizations corrected accordingly. To make sure that the correct sample drift is estimated, it is better to use multiple fiducial markers in the same sample, and the overall drift estimated from their individual drifts. However: (1) having multiple fiducials too close to the structure to be imaged will affect imaging and (2) the multiple fiducials might show different drift patterns, either due to movement within the sample or due to the variability of drift within the sample, and the overall drift estimation from them can be complicated.

In the case of imaging samples that have a clear structure, such as actin filaments or microtubules (as opposed to say, a sample with molecules distributed randomly in space), the subsets of localizations from adjacent frames lumped together might be correlated to each other throughout the imaging time. In this case, the information present in the correlation can be used to correct for drift. Multiple methods have been suggested to perform this correction [62,63].

\subsection{Fixation artifacts}

The long imaging time required for an SMLM experiment, necessitated by the need of imaging 1000s of frames, makes the use of SMLM for live cell imaging complicated. The use of fast imaging [64], including that of sCMOS cameras [65], and deconvolution based image processing algorithms [66] that allow higher density of imaged molecules per frame, have resulted in major improvements in this direction. However, the long imaging time is not an issue in the case of fixed samples, and most of the quantitative applications of PALM were done on them.

In the case of analysis that quantifies protein spatial distributions, or co-localization, it is important that the fixation preserves the protein configuration at the time of fixation. However, it has been found that after applying the fixative, different molecules get fixed at different time $[49,67]$. This means that the protein configuration is affected by fixation. Further studies are required to fully understand the effect of fixation artifacts on such quantitative studies.

\subsection{Representation}

SMLM experiments, after processing the raw data, provide a set of point localizations (estimates of the actual positions of the fluorescent tags) along with the estimated localization precision. Representing such information in an image format is a challenge, as merely representing the estimated locations as points is akin to overestimating the information available. Therefore the localization precision has also to be taken into consideration.

In the initial PALM papers [1], the researchers represented the data by means of a 'probability map', an image obtained by summing together Gaussian distributions corresponding to the localization uncertainty model estimated for each point localization, centered at the estimated localization. Such a representation involves loss in information. If $x_{A}, x_{B}, \ldots$ are true point locations of imaged molecules $A, B, \ldots$, then SMLM provides estimates of these locations $\widehat{x}_{A}, \widehat{x}_{B}$, . along with the error in estimation $\sigma_{\mathrm{A}}, \sigma_{\mathrm{B}}, \ldots$ In other words, probabilistic information about locations of individual molecules $-P(A)$, $P(B), \ldots$ - is available. In the case of a probability map representation, the available information is reduced to $P(A+B+\ldots)$, that is, there is information loss. However, it is possible to use the probability map as a worst case image.

Histogram based representation is another alternative that has been suggested. In this method, the estimated localizations are binned together. However, no information about estimation error is provided in this representation, and hence the method overestimates the available resolution. Additionally, binning can introduce artifacts to the representation and analysis, therefore the binning size must be carefully chosen.

Baddeley et al. have proposed alternative methods based on quad-tree and Delaunay triangulations [68]. However, all such representations also involve a loss of information compared to the raw results provided by SMLM. By the same reasoning, for quantitative analysis it is best to work with all the available information, i.e. the estimated locations and localization precision.

For analysis, researchers often select the localizations with the best precision for analysis, so as to obtain a more precisely localized structure. Such techniques can provide significant improvement in resolution [69]. However, since such a selection is a case of spatial sampling, the effect of it on analysis techniques must be addressed. The situation is more complicated if the distribution of localization precision in space is not homogeneous, since the selection then will be skewed. Such situations are possible due to non-homogeneous illumination, local variations in $\mathrm{pH}$ and even intermolecular interactions. Adequate precautions and controls must be done so as to ensure that artifacts are not created due to the use of a cut-off on localization precision.

\section{Conclusion}

In this article, we have presented a broad overview of the applications of SMLM in quantitative microscopy in varied fields of biology. We have also reviewed the various challenges that are present in using SMLM for quantitative measurements, with a focus on PALM, along with a brief review of the solutions that have been presented in the literature. Through cartoon figures, we have presented how the various errors that are present in the technique affect the three main ways SMLM data have been quantified: counting, cluster analysis and co-localization. We conclude that there is a critical need for accounting for these sources of errors, in order to achieve accurate and precise quantitative measurements. Furthermore, some of the challenges remain unsolved, and need novel solutions, both analytical and experimental.

\section{References}

[1] Betzig, E. Patterson, G.H. Sougrat, R, Lindwasser, O.W Olenych, S. Bonifacino, J.S., Davidson, M.W., Lippincott-Schwartz, J. and Hess, H.F. (2006) Imaging intracellular fluorescent proteins at nanometer resolution. Science 313, $1642-1645$.

[2] Hess, S.T., Girirajan, T.P.K. and Mason, M.D. (2006) Ultra-high resolution imaging by fluorescence photoactivation localization microscopy. Biophys. J . 91, 4258-4272.

[3] Rust, M.J., Bates, M. and Zhuang, X.W. (2006) Sub-diffraction-limit imaging by stochastic optical reconstruction microscopy (STORM). Nat. Methods 3, 793-795.

[4] Oddone, A., Vilanova, I.V., Tam, J. and Lakadamyali, M. (2014) Super-resolution imaging with stochastic single-molecule localization: concepts, technical developments, and biological applications. Microsc. Res. Tech..

[5] Klein, T., Proppert, S. and Sauer, M. (2014) Eight years of single-molecule localization microscopy. Histochem. Cell Biol., 1-15.

[6] Gould, T.J., Hess, S.T. and Bewersdorf, J. (2012) Optical nanoscopy: from acquisition to analysis. Annu. Rev. Biomed. Eng. 14. 10.1146/annurev-bioeng071811-150025. 
[7] Deschout, H., Shivanandan, A., Annibale, P., Scarselli, M. and Radenovic, A (2014) Progress in quantitative single-molecule localization microscopy. Histochem. Cell Biol.. 10.1007/s00418-014-1217-y.

[8] Lando, D., Endesfelder, U., Berger, H., Subramanian, L., Dunne, P.D., McColl, J. Klenerman, D., Carr, A.M., Sauer, M., Allshire, R.C., et al. (2012) Quantitative single-molecule microscopy reveals that CENP-ACnp1 deposition occurs during G2 in fission yeast. Open Biol. 2. 10.1098/rsob.120078.

[9] Renz, M., Daniels, B.R., Vámosi, G., Arias, I.M. and Lippincott-Schwartz, J. (2012) Plasticity of the asialoglycoprotein receptor deciphered by ensemble FRET imaging and single-molecule counting PALM imaging. PNAS. 10.1073/ pnas.1211753109.

[10] Nan, X., Collisson, E.A., Lewis, S., Huang, J., Tamguney, T.M., Liphardt, J.T., McCormick, F., Gray, J.W. and Chu, S. (2013) Single-molecule superresolution imaging allows quantitative analysis of RAF multimer formation and signaling. Proc. Natl. Acad. Sci. USA 110, 18519-18524. 10.1073/ pnas.1318188110.

[11] Gunzenhäuser, J., Olivier, N., Pengo, T. and Manley, S. (2012) Quantitative super-resolution imaging reveals protein stoichiometry and nanoscale morphology of assembling HIV-gag virions. Nano Lett. 12, 4705-4710. $10.1021 / \mathrm{nl} 3021076$

[12] Scarselli, M., Annibale, P. and Radenovic, A. (2012) Cell type-specific B2adrenergic receptor clusters identified using photoactivated localization microscopy are not lipid raft related, but depend on actin cytoskeleton integrity. J. Biol. Chem. 287, 16768-16780. 10.1074/jbc.M111.329912.

[13] Greenfield, D., McEvoy, A.L., Shroff, H., Crooks, G.E., Wingreen, N.S., Betzig, E. and Liphardt, J. (2009) Self-organization of the Escherichia coli chemotaxis network imaged with super-resolution light microscopy. PLoS Biol. 7. 10.1371/journal.pbio.1000137.

[14] Rossy, J., Owen, D.M., Williamson, D.J., Yang, Z. and Gaus, K. (2012) Conformational states of the kinase Lck regulate clustering in early $\mathrm{T}$ cell signaling. Nat. Immunol.. 10.1038/ni.2488.

[15] Sengupta, P., Jovanovic-Talisman, T., Skoko, D., Renz, M., Veatch, S.L. and Lippincott-Schwartz, J. (2011) Probing protein heterogeneity in the plasma membrane using PALM and pair correlation analysis. Nat. Methods 8, 969975. 10.1038/nmeth.1704.

[16] Annibale, P., Scarselli, M., Greco, M. and Radenovic, A. (2012) Identification of the factors affecting co-localization precision for quantitative multicolor localization microscopy. Opt. Nanoscopy 1. 10.1186/2192-2853-1-9.

[17] Pertsinidis, A., Mukherjee, K., Sharma, M., Pang, Z.P., Park, S.R., Zhang, Y. Brunger, A.T., Südhof, T.C. and Chu, S. (2013) Ultrahigh-resolution imaging reveals formation of neuronal SNARE/Munc18 complexes in situ. PNAS. 10.1073/pnas.1310654110.

[18] Lehmann, M., Rocha, S., Mangeat, B., Blanchet, F., Uji-i, H., Hofkens, J. and Piguet, V. (2011) Quantitative multicolor super-resolution microscopy reveals tetherin HIV-1 interaction. PLoS Pathog. 7. 10.1371/journal.ppat.1002456.

[19] Sherman, E., Barr, V., Manley, S., Patterson, G., Balagopalan, L., Akpan, I., Regan, C.K., Merrill, R.K., Sommers, C.L., Lippincott-Schwartz, J., et al. (2011) Functional nanoscale organization of signaling molecules downstream of the $\mathrm{T}$ cell antigen receptor. Immunity 35, 705-720, http://dx.doi.org/10.1016/ j.immuni.2011.10.004.

[20] Szymborska, A., de Marco, A., Daigle, N., Cordes, V.C., Briggs, J.A.G. and Ellenberg, J. (2013) Nuclear pore scaffold structure analyzed by superresolution microscopy and particle averaging. Science 341, 655-658, http:// dx.doi.org/10.1126/science.1240672.

[21] Loschberger, A., van de Linde, S., Dabauvalle, M.C., Rieger, B., Heilemann, M. Krohne, G. and Sauer, M. (2012) Super-resolution imaging visualizes the eightfold symmetry of gp210 proteins around the nuclear pore complex and resolves the central channel with nanometer resolution. J. Cell Sci. 125, 570 575, http://dx.doi.org/10.1242/Jcs.098822.

[22] Manley, S., Gillette, J.M., Patterson, G.H., Shroff, H., Hess, H.F., Betzig, E. and Lippincott-Schwartz, J. (2008) High-density mapping of single-molecule trajectories with photoactivated localization microscopy. Nat. Methods 5, 155-157, http://dx.doi.org/10.1038/nmeth.1176.

[23] Persson, F., Lindén, M., Unoson, C. and Elf, J. (2013) Extracting intracellular diffusive states and transition rates from single-molecule tracking data. Nat. Methods 10, 265-269, http://dx.doi.org/10.1038/nmeth.2367.

[24] Dempsey, G.T., Vaughan, J.C., Chen, K.H., Bates, M. and Zhuang, X. (2011) Evaluation of fluorophores for optimal performance in localization-based super-resolution imaging. Nat. Methods 8, 1027-1036, http://dx.doi.org/ 10.1038/nmeth.1768.

[25] Durisic, N., Laparra-Cuervo, L., Sandoval-Álvarez, Á., Borbely, J.S. and Lakadamyali, M. (2014) Single-molecule evaluation of fluorescent protein photoactivation efficiency using an in vivo nanotemplate. Nat. Methods, http://dx.doi.org/10.1038/nmeth.2784 (advance online publication).

[26] Thompson, R.E., Larson, D.R. and Webb, W.W. (2002) Precise nanometer localization analysis for individual fluorescent probes. Biophys. J . 82, 27752783, http://dx.doi.org/10.1016/S0006-3495(02)75618-X.

[27] Mortensen, K.I., Churchman, L.S., Spudich, J.A. and Flyvbjerg, H. (2010) Optimized localization analysis for single-molecule tracking and superresolution microscopy. Nat. Methods 7, 377-381, http://dx.doi.org/10.1038/ nmeth.1447.

[28] Annibale, P., Scarselli, M., Kodiyan, A. and Radenovic, A. (2010) Photoactivatable fluorescent protein mEos2 displays repeated photoactivation after a long-lived dark state in the red photoconverted form. J. Phys. Chem. Lett. 1, 1506-1510, http://dx.doi.org/10.1021/ Jz1003523.
[29] Annibale, P., Vanni, S., Scarselli, M., Rothlisberger, U. and Radenovic, A. (2011) Identification of clustering artifacts in photoactivated localization microscopy. Nat. Methods 8, 527-528.

[30] Lee, S.-H., Shin, J.Y., Lee, A. and Bustamante, C. (2012) Counting single photoactivatable fluorescent molecules by photoactivated localization microscopy (PALM). PNAS, http://dx.doi.org/10.1073/pnas.1215175109.

[31] Geisler, C., Hotz, T., Schönle, A., Hell, S.W., Munk, A. and Egner, A. (2012) Drift estimation for single marker switching based imaging schemes. Opt. Express 20, http://dx.doi.org/10.1364/OE.20.007274.

[32] Scarselli, M., Annibale, P., Gerace, C. and Radenovic, A. (2013) Enlightening Gprotein-coupled receptors on the plasma membrane using super-resolution photoactivated localization microscopy. Biochem. Soc. Trans. 41, 191-196, http://dx.doi.org/10.1042/Bst20120250.

[33] Scarselli, M., Annibale, P. and Radenovic, A. (2012) Cell type-specific beta2adrenergic receptor clusters identified using photoactivated localization microscopy are not lipid raft related, but depend on actin cytoskeleton integrity. J. Biol. Chem. 287, 16768-16780, http://dx.doi.org/10.1074/ jbc.M111.329912.

[34] Dani, A., Huang, B., Bergan, J., Dulac, C. and Zhuang, X. (2010) Superresolution imaging of chemical synapses in the brain. Neuron $68,843-856$, http:// dx.doi.org/10.1016/j.neuron.2010.11.021.

[35] MacGillavry, H.D., Song, Y., Raghavachari, S. and Blanpied, T.A. (2013) Nanoscale scaffolding domains within the postsynaptic density concentrate synaptic AMPA receptors. Neuron 78, 615-622, http://dx.doi.org/10.1016/ j.neuron.2013.03.009.

[36] Frost, N.A., Lu, H.E. and Blanpied, T.A. (2012) Optimization of cell morphology measurement via single-molecule tracking PALM. PLoS One 7, e36751, http:// dx.doi.org/10.1371/journal.pone.0036751.

[37] Sochacki, K.A., Larson, B.T., Sengupta, D.C., Daniels, M.P., Shtengel, G., Hess, H.F. and Taraska, J.W. (2012) Imaging the post-fusion release and capture of a vesicle membrane protein. Nat. Commun. 3, 1154, http://dx.doi.org/10.1038/ ncomms2158.

[38] Bar-On, D., Wolter, S., van de Linde, S., Heilemann, M., Nudelman, G., Nachliel, E., Gutman, M., Sauer, M. and Ashery, U. (2012) Super-resolution imaging reveals the internal architecture of nano-sized syntaxin clusters. J. Biol. Chem. 287, 27158-27167, http://dx.doi.org/10.1074/jbc.M112.353250.

[39] Yang, L., Dun, A.R., Martin, K.J., Qiu, Z., Dunn, A., Lord, G.J., Lu, W., Duncan, R.R. and Rickman, C. (2012) Secretory vesicles are preferentially targeted to areas of low molecular SNARE density. PLoS One 7, e49514, http://dx.doi.org/ 10.1371/journal.pone.0049514.

[40] Ptacin, J.L., Lee, S.F., Garner, E.C., Toro, E., Eckart, M., Comolli, L.R., Moerner, W.E. and Shapiro, L. (2010) A spindle-like apparatus guides bacterial chromosome segregation. Nat. Cell Biol. 12, 791-798, http://dx.doi.org/ $10.1038 /$ ncb2083.

[41] Pereira, C.F., Rossy, J., Owen, D.M., Mak, J. and Gaus, K. (2012) HIV taken by STORM: super-resolution fluorescence microscopy of a viral infection. Virol. J. 9, 84, http://dx.doi.org/10.1186/1743-422X-9-84.

[42] Lelek, M., Di Nunzio, F., Henriques, R., Charneau, P., Arhel, N. and Zimmer, C. (2012) Superresolution imaging of HIV in infected cells with FIAsH-PALM. Proc. Natl. Acad. Sci. USA 109, 8564-8569, http://dx.doi.org/10.1073/ pnas.1013267109.

[43] Wang, W., Li, G.W., Chen, C., Xie, X.S. and Zhuang, X. (2011) Chromosome organization by a nucleoid-associated protein in live bacteria. Science 333, 1445-1449, http://dx.doi.org/10.1126/science.1204697.

[44] Baday, M., Cravens, A., Hastie, A., Kim, H., Kudeki, D.E., Kwok, P.Y., Xiao, M. and Selvin, P.R. (2012) Multicolor super-resolution DNA imaging for genetic analysis. Nano Lett. 12, 3861-3866, http://dx.doi.org/10.1021/nl302069q.

[45] Deschout, H., Zanacchi, F.C., Mlodzianoski, M., Diaspro, A., Bewersdorf, J., Hess, S.T. and Braeckmans, K. (2014) Precisely and accurately localizing single emitters in fluorescence microscopy. Nat. Methods 11, 253-266, http:// dx.doi.org/10.1038/nmeth.2843.

[46] Small, A. and Stahlheber, S. (2014) Fluorophore localization algorithms for super-resolution microscopy. Nat. Methods 11, 267-279, http://dx.doi.org/ $10.1038 /$ nmeth.2844.

[47] Gould, T.J., Hess, S.T. and Bewersdorf, J. (2012) Optical nanoscopy: from acquisition to analysis. Annu. Rev. Biomed. Eng. 14, 231-254, http:// dx.doi.org/10.1146/annurev-bioeng-071811-150025.

[48] Ulbrich, M.H. and Isacoff, E.Y. (2007) Subunit counting in membrane-bound proteins. Nat. Methods 4, 319-321, http://dx.doi.org/10.1038/nmeth1024.

[49] Annibale, P., Scarselli, M., Greco, M. and Radenovic, A. (2012) Identification of the factors affecting co-localization precision for quantitative multicolor localization microscopy. Opt. Nanoscopy 1,9.

[50] Durisic, N., Godin, A.G., Wever, C.M., Heyes, C.D., Lakadamyali, M. and Dent, J.A. (2012) Stoichiometry of the human glycine receptor revealed by direct subunit counting. J. Neurosci. 32, 12915-12920, http://dx.doi.org/10.1523/ JNEUROSCI.2050-12.2012.

[51] Enderlein, J., Toprak, E. and Selvin, P.R. (2006) Polarization effect on position accuracy of fluorophore localization. Opt. Express 14, 8111-8120, http:// dx.doi.org/10.1364/Oe.14.008111.

[52] Stallinga, S. and Rieger, B. (2010) Accuracy of the Gaussian point spread function model in 2D localization microscopy. Opt. Express 18, 24461-24476, http://dx.doi.org/10.1364/OE.18.024461.

[53] Engelhardt, J., Keller, J., Hoyer, P., Reuss, M., Staudt, T. and Hell, S.W. (2011) Molecular orientation affects localization accuracy in superresolution far-field fluorescence microscopy. Nano Lett. 11, 209-213, http://dx.doi.org/10.1021/ nl103472b. 
[54] Churchman, L.S., Flyvbjerg, H. and Spudich, J.A. (2006) A non-Gaussian distribution quantifies distances measured with fluorescence localization techniques. Biophys. J. 90, 668-671, http://dx.doi.org/10.1529/ biophysj.105.065599.

[55] Ruprecht, V., Weghuber, J., Wieser, S. and Schütz, G.J. (2010) Chapter two measuring colocalization by dual color single molecule imaging: thresholds, error rates, and sensitivityAdvances in Planar Lipid Bilayers and Liposomes, pp. 21-40, Academic Press.

[56] Dickson, R.M., Cubitt, A.B., Tsien, R.Y. and Moerner, W.E. (1997) On/off blinking and switching behaviour of single molecules of green fluorescent protein. Nature 388, 355-358, http://dx.doi.org/10.1038/41048.

[57] McEvoy, A.L., Hoi, H., Bates, M., Platonova, E, Cranfill, P.J., Baird, M.A. Davidson, M.W., Ewers, H., Liphardt, J. and Campbell, R.E. (2012) MMaple: a photoconvertible fluorescent protein for use in multiple imaging modalities. PLoS One 7, http://dx.doi.org/10.1371/journal.pone.0051314. ARTN e51314.

[58] Annibale, P., Vanni, S., Scarselli, M., Rothlisberger, U. and Radenovic, A. (2011) Quantitative photo activated localization microscopy: unraveling the effects of photoblinking. PLoS One 6, e22678, http://dx.doi.org/10.1371/ journal.pone.0022678.

[59] Lee, S.H., Shin, J.Y., Lee, A. and Bustamante, C. (2012) Counting single photoactivatable fluorescent molecules by photoactivated localization microscopy (PALM). Proc. Natl. Acad. Sci. USA 109, 17436-17441, http:// dx.doi.org/10.1073/pnas.1215175109.

[60] Annibale, P., Vanni, S., Scarselli, M., Rothlisberger, U. and Radenovic, A. (2011) Identification of clustering artifacts in photoactivated localization microscopy. Nat. Methods 8, 527-528, http://dx.doi.org/10.1038/nmeth.1627.

[61] Pertsinidis, A., Zhang, Y.X. and Chu, S. (2010) Subnanometre single-molecule localization, registration and distance measurements. Nature 466, http:// dx.doi.org/10.1038/Nature09163 (647-U611).

[62] Geisler, C., Hotz, T., Schonle, A., Hell, S.W., Munk, A. and Egner, A. (2012) Drift estimation for single marker switching based imaging schemes. Opt. Express 20, 7274-7289, http://dx.doi.org/10.1364/OE.20.007274.

[63] Mlodzianoski, M.J., Schreiner, J.M., Callahan, S.P., Smolkova, K., Dlaskova, A., Santorova, J., Jezek, P. and Bewersdorf, J. (2011) Sample drift correction in 3D fluorescence photoactivation localization microscopy. Opt. Express 19, 15009-15019, http://dx.doi.org/10.1364/OE.19.015009.
[64] Jones, S.A., Shim, S.H., He, J. and Zhuang, X. (2011) Fast, three-dimensional super-resolution imaging of live cells. Nat. Methods 8, 499-508, http:// dx.doi.org/10.1038/nmeth.1605.

[65] Huang, F., Hartwich, T.M., Rivera-Molina, F.E., Lin, Y., Duim, W.C., Long, J.J., Uchil, P.D., Myers, J.R., Baird, M.A., Mothes, W., et al. (2013) Video-rate nanoscopy using sCMOS camera-specific single-molecule localization algorithms. Nat. Methods 10, 653-658, http://dx.doi.org/10.1038/ nmeth. 2488.

[66] Zhu, L., Zhang, W., Elnatan, D. and Huang, B. (2012) Faster STORM using compressed sensing. Nat. Methods 9, 721-723, http://dx.doi.org/10.1038/ nmeth.1978.

[67] Tanaka, K.A., Suzuki, K.G., Shirai, Y.M., Shibutani, S.T., Miyahara, M.S., Tsuboi, H., Yahara, M., Yoshimura, A., Mayor, S., Fujiwara, T.K., et al. (2010) Membrane molecules mobile even after chemical fixation. Nat. Methods 7, 865-866 http://dx.doi.org/10.1038/nmeth.f.314.

[68] Baddeley, D., Cannell, M.B. and Soeller, C. (2010) Visualization of localization microscopy data. Microsc. Microanal. 16, 64-72, http://dx.doi.org/10.1017/ S143192760999122x.

[69] Pertsinidis, A., Mukherjee, K. Sharma, M., Pang, Z.P.P., Park, S.R., Zhang, Y.X Brunger, A.T., Sudhof, T.C. and Chu, S. (2013) Ultrahigh-resolution imaging reveals formation of neuronal SNARE/Munc18 complexes in situ. Proc. Natl. Acad. Sci. USA 110, E2812-E2820, http://dx.doi.org/10.1073/ pnas.1310654110.

[70] Wang, S., Moffitt, J.R., Dempsey, G.T., Xie, X.S. and Zhuang, X. (2014) Characterization and development of photoactivatable fluorescent proteins for single-molecule-based superresolution imaging. Proc. Natl. Acad. Sci. USA 111, 8452-8457, http://dx.doi.org/10.1073/pnas.1406593111.

[71] Ianoul, A., Grant, D.D., Rouleau, Y., Bani-Yaghoub, M., Johnston, L.J. and Pezacki, J.P. (2005) Imaging nanometer domains of beta-adrenergic receptor complexes on the surface of cardiac myocytes. Nat. Chem. Biol. 1, 196-202, http://dx.doi.org/10.1038/nchembio726.

[72] Holden, S.J., Pengo, T., Meibom, K.L., Fernandez, C., Collier, J. and Manley, S. (2014) High throughput 3D super-resolution microscopy reveals Caulobacter crescentus in vivo Z-ring organization. Proc. Natl. Acad. Sci. USA 111, 45664571, http://dx.doi.org/10.1073/pnas.1313368111. 